\title{
Model of the frost deformation in thermoplastic-photoconductor films
}

\author{
F. Carreño and E. Bernabeu \\ Departamento de Optica, Facultad de Ciencias Físicas, Universidad Complutense, 28040 Madrid, Spain
}

(Received 2 July 1993; accepted for publication 24 January 1994)

The dynamics of thermoplastic-photoconductor films is reviewed. A theory of thermoplastic film deformation is presented for a three-layer structure in air with modified boundary conditions. The amplitude of the deformation $A$ versus the spatial frequency $k$ is of bandpass type ( $A-k$ curve). The influence of the thickness of the layers and the heating and electric parameters on the $A-k$ curve are analyzed. The predictions of the model are compared to others previously reported and with experimental data. The validity and limitations of the model are also discussed.

\section{INTRODUCTION}

Thermoplastic-photoconductor materials for recording optical information are well known. ${ }^{1-6}$ They are considered very promising candidates for the storage of optical signals $\mathrm{s}^{7,8}$ and for nondestructive testing by holographic interferometry. ${ }^{9}$

The frost effect presentcd by these materials is related to spontaneous deformations observed when the charged films are heated to a temperature close to the glass transition $T_{g}$, where they become fluid. ${ }^{10} \mathrm{~A}$ first approach to this problem was presented in Budd's article, ${ }^{11}$ where a dispersion relation between the rate of growth of deformation and spatial frequency was obtained. Budd used an effective surface stress by subtracting from the surface stress a term related to the electrostatic energy of the layer and considering only the influence of the forces normally oriented. An improvement of Budd's theory was presented by Killat ${ }^{12}$ for a single layer, considering the effects of the electrostatic polential inside the film in the boundary conditions, i.e., in the thermoplastic and air. The performance and limitations of these films are also analyzed by Killat and Terrel in a subsequent article. ${ }^{13}$ The most important criticism concerning the models derived by Budd and Killat is that they consider a single layer: This is not realistic, since the thermoplastic film is limited, at least, by a photoconductive layer and, optionally, by a dielectric substrate. Another important limitation of these models is that they consider that there is no charge leakage during the heating but, near $T_{g}$, charge decay should be assumed to take place due to the finite value of the thermal conductivity of the thermoplastic. This latter assumption is considered by Hirshfeld, Friesem, and Rav-Noy ${ }^{14}$ but, on the contrary, they do not take into account the effects of the electrostatic field distribution inside the film, in spite of the fact that they explicitly state that the electric forces are responsible for the growth of the deformation. A different approach is due to Handojo, ${ }^{15}$ explaining the frost deformation from thermodynamic and electrostatic considerations.

The purpose of this work is to show from a hydrodynamic point of view the importance of the electric parameters involved. A dispersion relation for the rate of growth of the deformation and the spatial frequency is obtained. This relation reduces to Budd's results when the electric effects are negligible. The amplitude of the deformation is also ob- tained as a function of the spatial frequency, the heating parameters, and the electric conditions.

It is very important to understand the underlying mechanisms and the relative importance of the different parameters involved for the design and optimization of thermoplastic materials.

Section II is devoted to the introduction of the basic equations; in Sec. III the generalizcd boundary conditions are obtained, and the predictions are presented in Sec. IV. The limitations of the model and the conclusions are presented in Sec. V.

\section{THE MOTION OF A VISCOUS FLUID}

The three-layer structure we consider is schematized in Fig. 1. The thermoplastic is considered as a viscous fluid bounded by a solid photoconductive layer at $y=h_{1}$ and air at $y=h_{2}$. Both the photoconductor and the thermoplastic are coated over a plastic substrate at $y=h_{0}$. The substrate is in contact with a grounded transparent plate. In the usual recording procedure the thermoplastic surface is charged by a corona device (tips or wires kept under high voltage around $10 \mathrm{kV}$ ). Then the system may be exposed to a monochromatic optical pattern that will generate a latent electrostatic image. Development is carried out heating the thermoplastic close to the glass transition temperature $T_{g}$ which enables us to fix the optical pattern as variations of the thickness of the thermoplastic layer (phase grating). Readout is accomplished after cooling by illuminating the system with monochromatic light of the same or different wavelength employed during exposure. We assume that the heating will not modify the optical properties of the thermoplastic layer (mainly the refraction index), thus the phase shift introduced by the grating is mainly due to thickness variatiors.

The glass transition is characterized by a diminished viscosity that allows the thermoplastic to flow according to the electric forces. This effect is accompanied by an increased conductivity. ${ }^{16}$ It is reasonable to assume that the increased conductivity is followed by a decay of the electric field. We will show that calculations based on decaying electric field taking into account appropriate boundary conditions are able to explain many experimental observations.

The flow field is considered to have an $x-y$ dependence (bidimensional flow field), and $v_{x}$ and $v_{y}$ are, respectively, the $x$ and $y$ components of the velocity $\mathbf{v}$. We shall look for 


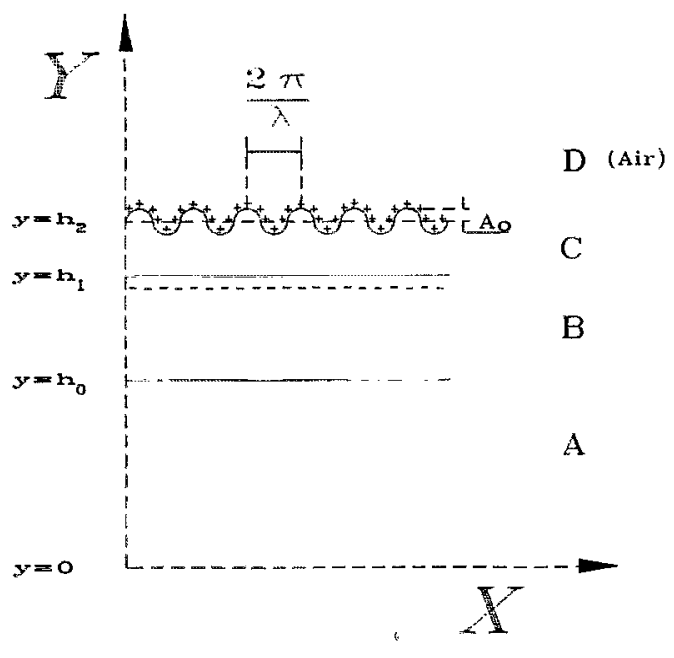

FIG. 1. Coordinate system of the problem. $h=h_{2}-h_{1}$ is the thickness of the thermoplastic layer, $k=2 \pi / \lambda$ is the spatial frequency of the deformation, and $A_{0}$ is the amplitude of the deformation.

an harmonic solution of the surface displacement of the form

$$
s(x, t)=A(t) \cos (k x),
$$

where the amplitude $A(t)$ may be written as

$$
A(t)=A_{0} \exp (\omega t)+A_{1} \exp (-\Omega t),
$$

where $\omega$ is the rate of growth of the deformation and $\Omega$ is the charge decay rate and may be found by modeling the thermoplastic layer as a parallel-plane capacitor to be twice the charge-decay rate,

$$
\Omega=2 \sigma / \epsilon_{0} \epsilon_{c},
$$

where $\sigma$ is the thermal conductivity of the thermoplastic and $\epsilon_{\mathrm{c}}$ is the diclectric constant of this layer in units of $\epsilon_{0}$. At $t=0$ there is no deformation, thus $A_{1}=-A_{0}$.

The Navier-Stokes equation for an incompressible fluid $(\nabla \cdot v=0)$ is considered to describe the movement of the fluid, ${ }^{17}$

$$
\rho\left(\frac{\partial \mathbf{v}}{\partial t}+(\mathbf{v} \cdot \nabla) \mathbf{v}\right)=-\nabla p+\eta \nabla^{2} \mathbf{v}
$$

where $\rho$ and $\eta$ are the density and the viscosity of the fluid respectively, and $p$ stands for pressure. In the following analysis $h$ stands for $h_{2}-h_{1}$ and is the thickness of the thermoplastic layer. An extra difficulty appears with the convection term $(\mathbf{v} \cdot \nabla) \mathbf{v}$ in Eq. (2.4) in order to find an analytical solution. This tcrm can be neglected if the Reynolds number $(\mathscr{B})$ is small enough. ${ }^{17}$ We assume that $\mathscr{R} \ll 1$ and then Eq. (2.4) becomes

$$
\frac{\partial \mathbf{v}}{\partial t}=-\frac{1}{\rho} \nabla p+\nu \nabla^{2} \mathbf{v}
$$

where $\nu \equiv \eta / \rho$ is the kinematics viscosity. The effects of induced dipolar forces due to the surface charge distribution are not taken into account. Gravitational forces are also ex- cluded in this analysis. The coefficients $\rho$ and $\nu$ are considered constants (independent of time) in the following analysis.

\section{GENERALIZED BOUNDARY CONDITIONS}

The photoconductive layer is supposed to have an infinite viscosity. An adherence effect in the layer near the fluid and the surface of the fixed solid (the photoconductor) is produced due to the attracting molecular forces, so in this boundary

$$
\mathbf{v}\left(y=h_{1}\right)=\mathbf{0} \text {. }
$$

The velocity of a moving boundary must be equal to the surface velocity, ${ }^{17}$ therefore $v_{y}$ is identified with the temporal variation of the deformation of the surface

$$
v_{y}=\frac{\partial s}{\partial t} .
$$

When the fluids are not mixed, the forces between them are equal and acting in contrary directions; this condition can be written as

$$
n_{1, k} \sigma_{1, j k}^{h}+n_{2, k} \sigma_{2, j k}^{h}=0,
$$

where the subscripts 1 and 2 apply to each fluid (inside and outside the thermoplastic respectively) and $n_{1}$ and $n_{2}$ stand for the normal vectors at the surface of each medium. In Eq. (3.3) $\sigma_{j k}^{h}$ stands for the stress tensor, which for an incompressible fluid can be expressed as

$$
\sigma_{j k}^{h}=-p \delta_{j k}+\eta\left(\frac{\partial v_{j}}{\partial x_{k}}+\frac{\partial v_{k}}{\partial x_{j}}\right),
$$

where $\delta_{j k}$ is the Krönecker tensor and the subscript $j=1,2$ stands for $x$ and $y$, respectively. When electrical and superficial stress forces are present, Eq. (3.3) is expressed as

$$
\sum_{k} n_{k}\left(\sigma_{1, j k}^{h}+\sigma_{1, j k}^{\mathrm{el}}-\sigma_{2, j k}^{h}-\sigma_{2, j k}^{\mathrm{el}}\right)=T \frac{\partial^{2} s}{\partial x^{2}} n_{i},
$$

$T$ being surface tension, and $\sigma_{j k}^{\mathrm{el}}$ is the Maxwell stress tensor given by

$$
\sigma_{j k}^{\mathrm{el}}=+\epsilon_{0} \epsilon\left(E_{j} E_{k}-\frac{1}{2} E^{2} \delta_{j k}\right)
$$

It was taken into account that $n_{1, j}=-n_{2, j}$ to deduce Eq. (3.5). The model of Budd ${ }^{11}$ assumes a zero tangential stress. In this case we have considered a nonzero tangential stress due to the presence of the electrostatic forces. If we consider that the surface pressure arises from the modulation of the electric field in the thermoplastic layer, at the temperature at which the thermoplastic is soft enough to flow $T_{g}$, a charge decay takes place. This process is described as a decaying sinusoidal surface pressure of the form

$$
\bar{p}=p_{0} \cos (k x) \exp (-\Omega t),
$$

where $p_{0}$ is the amplitude of the surface pressure and $\Omega$ is given by Eq. (2.3).

A model of the fields inside the different layers must be considered to evaluate the Maxwell stress tensor. The expres- 
sions derived for the field inside and outside the thermoplastic in the Appendix are used for this purpose. Equations (3.5) become

$$
\begin{aligned}
& \sigma_{1, x y}^{h}+\sigma_{0} E_{t D}=\sigma_{2, x y}^{h}, \\
& \sigma_{1, y y}^{h}+\frac{1}{2}\left\{C_{0}^{2}+2 C_{0} k\left[C_{1} \exp \left(k h_{2}\right)-C_{2}\right.\right. \\
& \left.\left.\quad \times \exp \left(-k h_{2}\right)\right] s(x, t)\right\}=\sigma_{2, y y}^{h}+T \frac{\partial^{2} s(x, t)}{\partial x^{2}}+\bar{p},
\end{aligned}
$$

where $E_{t D}$ stands for the tangential field at the top of the thermoplastic layer $y=h_{2}$ and $\sigma_{0}, C_{0}, C_{1}$, and $C_{2}$ are given in the Appendix. The nonlinear terms in $s^{2}(x, t)$ that would produce higher harmonics were neglected to deduce Eq. (3.8). The main difference between equation (3.8) and that derived by Killat $^{12}$ is that we have taken into account the structure of the film to obtain the electrostatic field distribution inside the film (see Appendix) and the decaying surface pressure $\bar{p}$. Equation (2.5) and the boundary conditions given by Eq. (3.1), (3.2), and (3.8) may be solved, ${ }^{18}$ and the following dispersion relation is obtained:

$f(S, k h)+\frac{T}{\eta}+\frac{\sigma_{0}}{\eta k}\left[C_{1} \exp \left(k h_{2}\right)-C_{2} \exp \left(-k h_{2}\right)\right]=0$

where $S=\left[1+\left(\omega / \nu k^{2}\right)\right]^{1 / 2}$ and $f(S, k h)$ is given by

$$
\begin{aligned}
f(S, k h)= & \left(S^{2}-1\right)\left[T_{00} S \cosh (k h)+T_{11} \sinh (k h)\right] \\
& +2\left\{T_{00} S[\cosh (k h)-\cosh (S k h)]\right. \\
& \left.+T_{11}[\sinh (k h)-S \sinh (S k h)]\right\}
\end{aligned}
$$

and the amplitude of the deformation is given by

$$
A_{0}=-\frac{p_{0} / \eta k^{2}}{f\left\{\left[1+\left(\Omega / \nu k^{2}\right)\right]^{1 / 2}, k h\right\}+(T / \eta)+\left(\sigma_{0} / \eta k\right)\left[C_{1} \exp \left(k h_{2}\right)-C_{2} \exp \left(-k h_{2}\right)\right]} .
$$

In Eq. (3.10) $T_{00}$ and $T_{11}$ are given by

$$
\begin{aligned}
& T_{00}=\frac{\nu k\left(S^{2}-1\right) T_{3}-T_{1} T_{4}}{\left(S^{2}-1\right)[S \cosh (S k h) \sinh (k h)-\sinh (S k h) \cosh (k h)]}, \\
& T_{11}=\frac{T_{0} T_{4}-\nu k\left(S^{2}-1\right) T_{2}}{\left(S^{2}-1\right)[S \cosh (S k h) \sinh (k h)-\sinh (S k h) \cosh (k h)]},
\end{aligned}
$$

and $T_{0}, T_{1}, T_{2}, T_{3}$, and $T_{4}$ stand for

$$
\begin{aligned}
& T_{0}=S \sinh (k h)-\sinh (S k h), \quad T_{1}=\cosh (k h)-\cosh (S k h), \quad T_{2}=\left(S^{2}+1\right) \sinh (S k h)-2 S \sinh (k h), \\
& T_{3}=\left(S^{2}+1\right) \cosh (S k h)-2 \cosh (k h), \quad T_{4}=\frac{\sigma_{0} D_{2} \exp \left(-k h_{2}\right)}{\eta k} .
\end{aligned}
$$

In the limit of a high viscous medium, Eq. (3.10) can be expanded in powers of $\omega / \nu k^{2}$, and retaining the first nonzero term we finally obtain

$$
\omega=\frac{6 \nu k^{2}\left\{-(T / \eta)-\left(\sigma_{0} / \eta k\right)\left[C_{1} \exp \left(k h_{2}\right)-C_{2} \exp \left(-k h_{2}\right)\right]\left[\frac{1}{2} \sinh (2 k h)-k h\right]-\frac{1}{2} T_{4}(k h)^{2}\right\}}{2 a_{3}},
$$

where $a_{3}$ is given by

$$
a_{3}=\frac{3}{8}\left[8 k \nu-T_{4}+16 k \nu(k h)^{2}+2 T_{4}(k h)^{2}+8 k \nu \cosh (2 k h)+T_{4} \cosh (2 k h)\right] .
$$

The solution obtained by Budd for $\omega(k)$ is recovered in the limit of $\sigma_{0} \rightarrow 0$ in Eq. (3.14). The amplitude of the deformation in the high viscous approximation can be expressed as

$A_{0}=\frac{-p_{0}}{\eta k^{2}\left(\left\{T_{4}(k h)^{2}+a_{3} \Omega / 3 \nu k^{2}\right\} /\{[\sinh (2 k h) / 2]-k h\}+(T / \eta)+\left(\sigma_{0} / \eta k\right)\left[C_{1} \exp \left(k h_{2}\right)-C_{2} \exp \left(-k h_{2}\right)\right]\right)}$.

\section{PREDICTIONS OF THE MODEL}

In Fig. 2 we have plotted the rate of growth for several values of $\sigma_{0}$. The dashed line give the results obtained without considering electrostatic forces (as in Ref. 14). It is obvious from Fig. 2 that the electric-field distribution tends to reinforce medium spatial frequencies. In the limit of high spatial frequencies the results of the model tends asymptotically to the values predicted by other models.
The previously mentioned articles, except Ref. 14, focus attention on the dispersion relation $\omega(k)$, whereas the main parameters in holography are the amplitude of the deformation $A$ versus the spatial frequency $k$ curve which we shall call the $A-k$ curve and its bandwidth. The sensitivity of the material with its rheological properties, the heating parameter, the electrical characteristics, and the thickness of the different layers must be analyzed. 


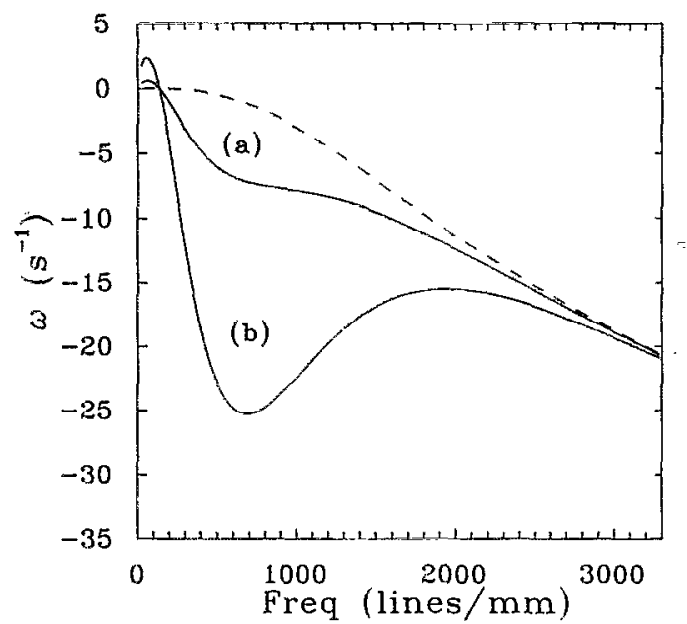

FIG. 2. Growth rate $\omega$ as a function of spatial frequency: (a) $\sigma_{0}=0.03 \mathrm{C} / \mathrm{m}^{2}$ and (b) $\sigma_{0}=0.06 \mathrm{C} / \mathrm{m}^{2}$. The dashed line is predicted by the Hirshfeld model. $\epsilon_{c}=2.3$ is used for the thermoplastic layer as it is appropriate to Staybelite Ester 10, a commonly used thermoplastic.

The data used in subsequent calculations are $\rho=1000$ $\mathrm{kg} / \mathrm{m}^{2}, T=0.01 \mathrm{~N} / \mathrm{m}^{2}, \eta=10^{6} \mathrm{P}, p_{0}=10^{6} \mathrm{~N} / \mathrm{m}^{2}$, and the dielectric constants of the layers A, B, C, and D were, respectively, $\epsilon_{a}=2, \epsilon_{b}=3, \epsilon_{c}=2.3$ and $\epsilon_{d}=1$ (air). The dielectric constants are in units of the vacuum dielectric constant $\epsilon_{0}$.

\section{A. Heat-development energy}

The heat-development energy enters in the model affecting $\eta$ and $\sigma$. For the temperatures near or above the glass transition temperature $T_{g}$, the following approximate relation holds: ${ }^{16}$

$v \eta=$ const.

In the rigid state, i.e., while the viscosity is high, the deformation amplitude $A(t)$ is maintained constant. The duration of this state is given approximately by the time required to approach to a temperature close to $T_{g}\left(\Delta t_{0}\right)$ where the thermoplastic becomes fluid. The value of $\Delta t_{0}$ depends mainly on the thickness of the film, the thermal diffusivity, and the heating power. ${ }^{19}$

If we consider a fluid state during a certain time $\Delta t$, the process can be described as

$A(t)=\left\{\begin{array}{l}0, \quad t \leqslant \Delta t_{0}, \\ A_{0}[\exp (-\Omega t)-\exp (\omega t)], \quad \Delta t_{0} \leqslant t \leqslant \Delta t, \\ A_{0}[\exp (-\Omega \Delta t)-\exp (\omega \Delta t)] ; \quad t \geqslant \Delta t,\end{array}\right.$

i.e., there is no deformation during the rigid state, then it starts to grow, reaches a maximum and, optionally, decreases until it stops at $t=\Delta t$. It is clear from Eq. (4.2) that the terms in $\Omega \Delta t$ and in $\omega \Delta t$ increase or decrease by the same factor, while $A_{0}$ given by Eq. (3.16) is unaffected; thus, $\Omega \Delta t$ should be a candidate as a parameter for characterizing the development.

The $A-k$ curve as a function of the heating parameter is shown in Fig. 3. When $\Omega \Delta t \ll 1$ the deformation remains

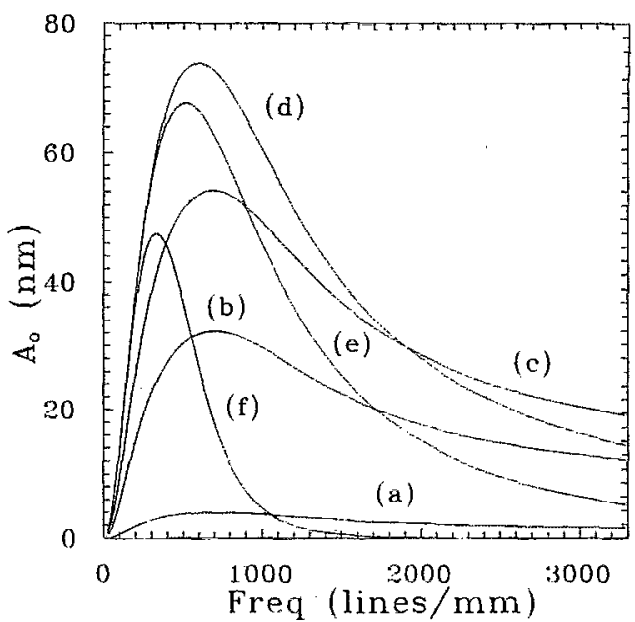

FIG. 3. Theoretical deformation amplitude vs spatial frequency ( $A-k$ curve) for different heating parameters $\Omega \Delta t$ for $h=0.25 \mu \mathrm{m}$ : (a) $\Omega \Delta t=0.05$; (b) $\Omega \Delta t=0.5$; (c) $\Omega \Delta t=1.1$; (d) $\Omega \Delta t=5.0$; (e) $\Omega \Delta t=10.0$; (f) $\Omega \Delta t=50.0$.

almost constant for all spatial frequencies and close to zero, i.e., it is underdeveloped. The typical bandpass response appears when the value of the heating parameter is increased and for $\Omega \Delta t \gg 1$ the deformation is reduced again. This last result is due to the reduction of the internal stress of the material to minimum values: Because of this the material can be reused. The working point should be selected in the intermediate region where the width and the amplitude are adequate for the recording of holograms. We note here that the diffraction efficiency (DE) of a thin two-dimensional sinusoidal phase grating is given by ${ }^{20}$

$$
\mathrm{DE}=J_{1}^{2}(B),
$$

where $J_{1}(B)$ is the Bessel function of first order and $B$ is given by

$$
B=\frac{2 \pi}{\lambda}(n-1) A_{m},
$$

$\lambda$ being the wavelength employed for the recording-readout of the holograms, $n$ the refractive index of the thermoplastic layer, and $A_{m}$ the depth of the deformation. Figure 4 shows the $\mathrm{DE}$ as a function of the heating parameter for two values of the thermoplastic thickness $h$. There is an optimum heat to get the maximum DE for a fixed value of $h$. It should be noted that for low values of $h$, the DE of the holograms will be reduced in comparison to that obtained for larger values. It becomes clear now the assumption mentioned in Sec. II related to the constant value of the refraction index of the thermoplastic layer: If both $n$ and the thickness of the thermoplastic layer change it will produce a mixed grating, which is considered undesirable for the recording of holograms. ${ }^{20}$

\section{B. Influence of electrostatic forces}

The effect of surface charge density in the $A-k$ curve is shown in Fig. 5: The tangential electric stress increases when $\sigma_{0}$ is increased, producing a reduction of the peak deforma- 


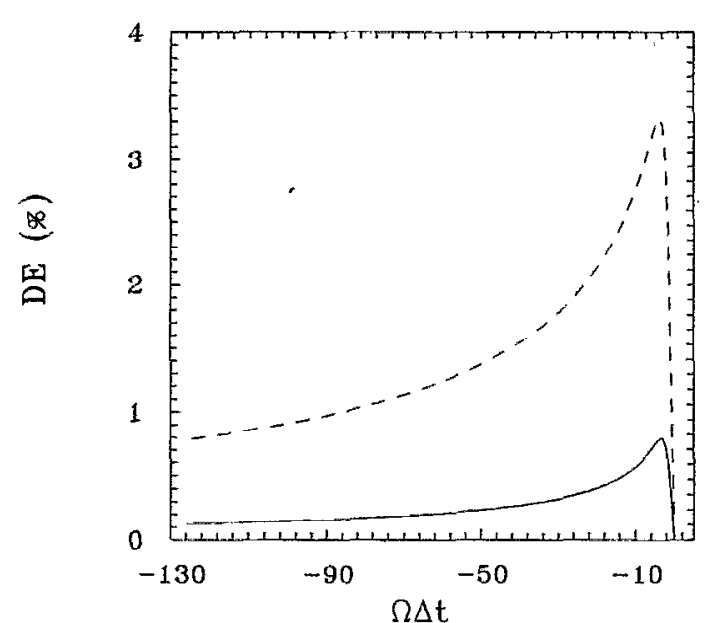

FIG. 4. Diffraction efficiency $\mathrm{DE}$ as a function of the heating parameter $\Omega \Delta t$. Continuous line: for $h=0.25 \mu \mathrm{m}$; dashed line: $h=0.50 \mu \mathrm{m}$.

tion, and a shift of this peak to lower spatial frequencies. The effect on the DE should also be considered due to the contrary effects of heating and charging. Figure 6 shows a 3D plot of the peak deformation as a function of hcating and charging parameters for $h=0.25 \mu \mathrm{m}$.

The effects on the resolution (bandwidth) and DE efficiency of the surface charge density are less pronounced for low values of the thermoplastic thickness, i.e., thermoplastic films of reduced thickness (around $0.20 \mu \mathrm{m}$ ) are less sensitive to variations of charging conditions: This is very important because these materials are usually sensitized using corona devices and, due to the random nature of the Townsend discharge, it is difficult to reproduce the same conditions during sensitization of different frames unless considerable charging times (around $200 \mathrm{~s}$ ) are used. ${ }^{21}$

The influence of the thermoplastic dielectric constant in the $A-k$ curve is of second order. This result is contrary to that reported by Killat, ${ }^{12}$ as the model employed there attrib-

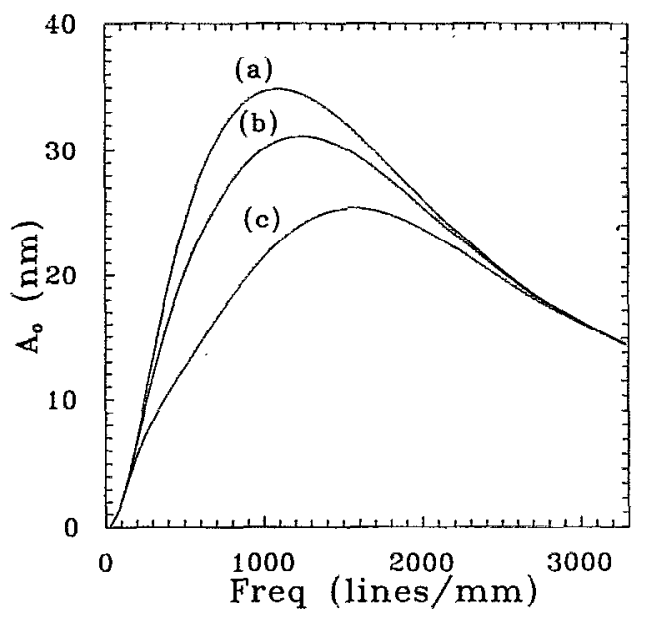

FIG. 5. Deformation amplitude vs spatial frequency ( $A-k$ curve) for different values of the surface charge density: (a) $\sigma_{0}=0.0001 \mathrm{C} / \mathrm{m}^{2}$; (b) $\sigma_{0}=0.03$ $\mathrm{C} / \mathrm{m}^{2} ;(c) \sigma_{0}=0.06 \mathrm{C} / \mathrm{m}^{2}$.

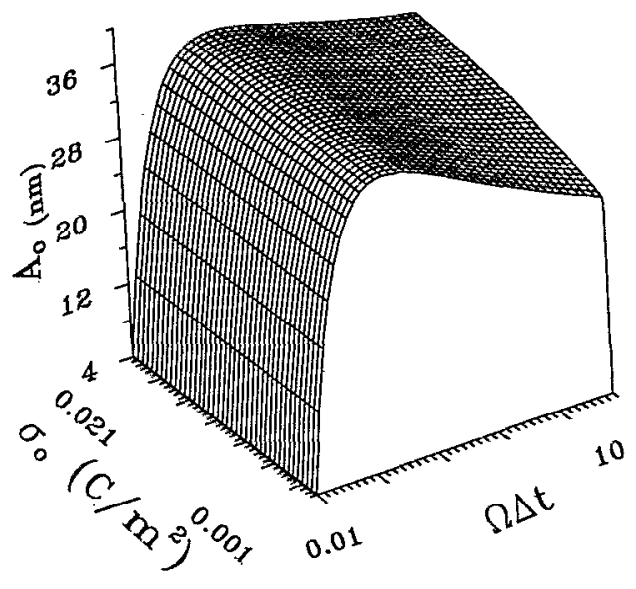

FIG. 6. 3D plot of the theoretical maximum deformation produced as a function of the heating parameter $\Omega \Delta t$ and the surface charge density.

uted the effect to the thermoplastic, but according to Schaffert, ${ }^{22}$ the main layer in electric terms is the photoconductive layer. When the dielectric constant of the photoconductive layer is increased, the amplitude of the deformation reduces its magnitude.

Figure 7 shows the dependence of peak deformation with charging conditions and fixed power heating. The values have been determined with an atomic force microscope (NanoScope from Digital Instruments) working in tapping mode. The peak deformation increases when the high voltage applied to the corona unit (and then the value of $\sigma_{0}$ available) is increased. The value of the peak deformation is in the range of $10-80 \mathrm{~nm}$ which remains within the limits of application of the model $(98 \ll 1)$. Now the influence of the electrostatic forces becomes clear to properly describe the dynamics of thermoplastic films.

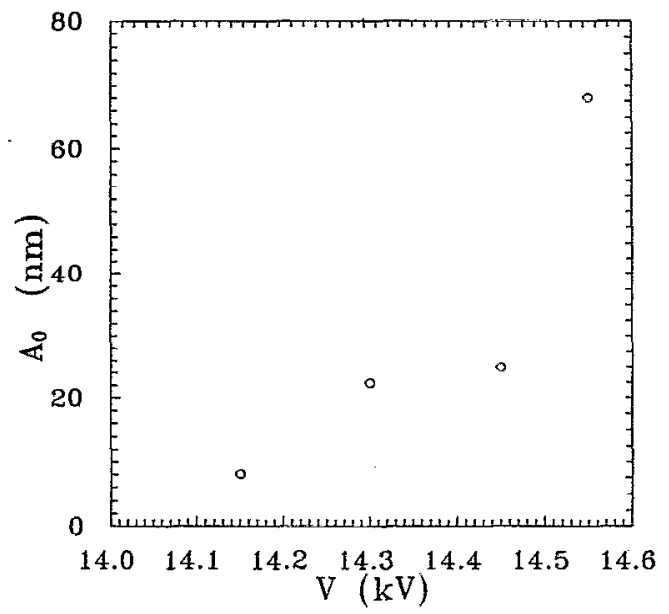

FIG. 7. Maximum deformation achieved for different charging conditions. $V$ is the high voltage applied to the corona device. The probe scanned an area of $100 \mu \mathrm{m}^{2}$. 


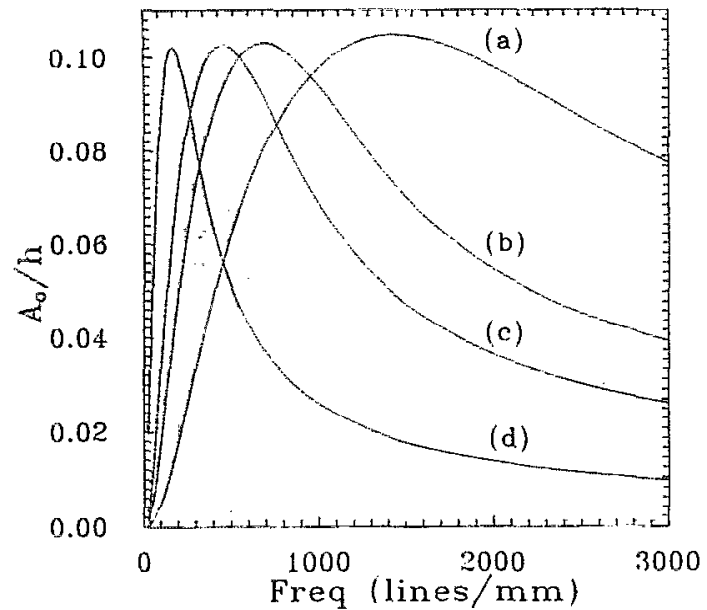

FIG. 8. Theoretical $A-k$ curve for different values of $h$. The curves were divided by the thickness of the thermoplastic layer for comparison purposes. (a) $h=0.25 \mu \mathrm{m}$, (b) $h=0.50 \mu \mathrm{m}$, (c) $h=1.0 \mu \mathrm{m}$, (d) $h=2.0 \mu \mathrm{m}$.

\section{Thickness of the layers}

The effects of the thickness of the different layers are described in the following. The substrate is used as a physical support of the other layers: Its effects on the dynamics have becn analyzed by us using Eqs. (3.14)-(3.16), showing that the influence of the substrate parameters $\left(\epsilon_{a}\right.$ and $\left.h_{0}\right)$ are of second order.

The role of the photoconductor is mainly to keep the charges in the absence of light and to allow a selective migration of charges during the exposure to both object and reference beams during the recording of holograms. The electrostatic latent image can be fixed later when the film is heated. A low dark current is required for this layer to be useful for the recording of holugrams, then a high value of the dielectric constant is required.

The central layer is the thermoplastic. The effect of changing the thickness of this layer $h$, is of major importance: When it is increased the bandwidth decreases drastically while the peak deformation is increased. Figure 8 shows the typical bandpass response of these materials for different values of $h$. Figure 9 shows the spatial frequency at which the peak deformation is achieved $f_{\max }$ as a function of the thickness. In each one of the figures the surface charge was maintained constant while the heating parameter was varied from conditions close to underdevelopment to overdevelopment. A nonlinear behavior appears for low values of the heating parameter $\Omega \Delta t$ and tends to a linear relation for higher values of $\Omega \Delta t$. The influence of the electrostatic forces is to increase $f_{\max }$ to higher spatial frequencies when $\Omega \Delta t$ is low, whereas when the heating is strong the electrostatic forces tend to give a soft dependence for $f_{\max }$. with $h$. This effect is better appreciated for high values of the surface charge density (see Fig. 10) with deviations around $40 \%$ with regard to models that neglect the influence of these forces.

\section{Comparison with experimental data}

The model proposed for the dynamical evolution of the surface displacement given by Eq. (4.2) is of more precise

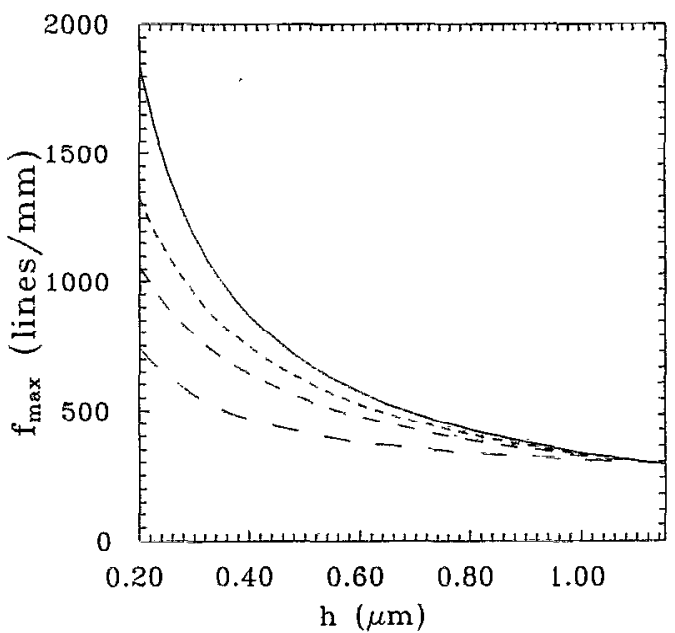

FIG. 9. Theoretical spatial frequency $f_{\max }$ at which the maximum deformation is produced as a function of the thermoplastic layer thickness for different values of the heating parameter. The dashed lines are put in order of increasing values of $\Omega \Delta t=5,10,30$, and the continuous line is for $\Omega \Delta t=1$. $\sigma_{0}=0.001$ for all of them.

nature than that given in Ref. 14 as it incorporates a new parameter $\Delta t_{0}$, which is related to the duration of the rigid state. We have tried to verify the validity of this model from an experimental viewpoint using the setup proposed in Ref. 23. The three-layer film employed was a commercial available thermoplastic film. The film was illuminated by an IR emitter (SFH 400-3) and the light scattered by the surface out of the specular angle was collected by an IR photodetector (BPX 43-3). The photosignal was amplified and stored in a digital scope HP54501 A and transferred to a computer for later analysis. The photosignal can be related to the surface displacement by computing the scattered electric field by a slow moving boundary using standard techniques. ${ }^{20}$ Figure

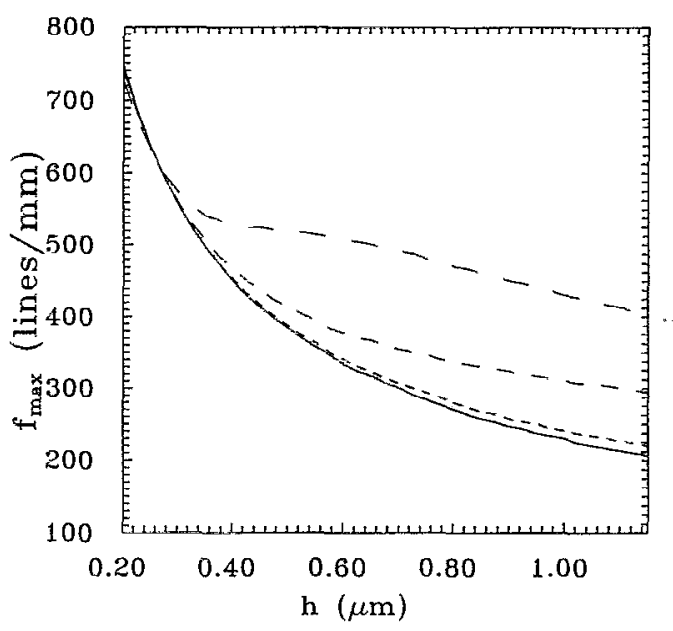

FIG. 10. Theoretical spatial frequency $f_{\max }$ at which the maximum deformation is produced as a function of the thermoplastic layer thickness $h$ for different values of the surface charge density. The dashed lines are put in order of increasing values of $\sigma_{0}=0.004,0.009,0.015$, and the continuous line is for $\sigma_{0}=0.001 . \Omega \Delta t=30$ for all of them. 


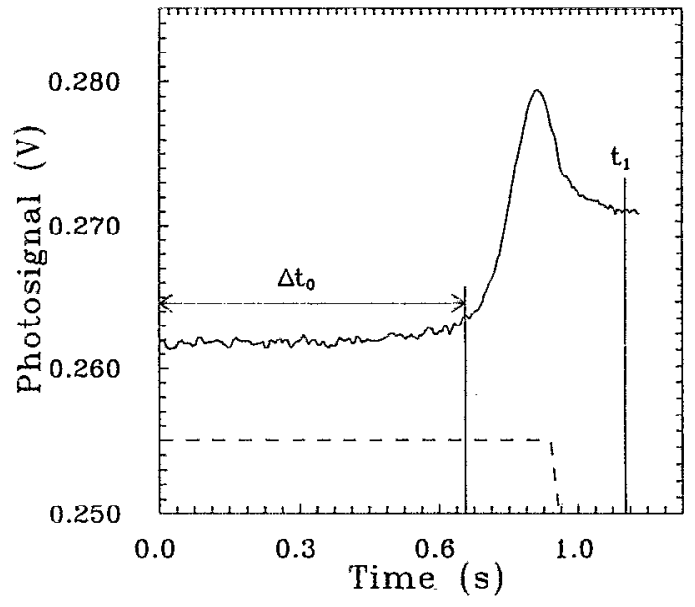

FIG. 11. Photosignal (continuous line) and heating pulse (dashed line). The heating pulse is scaled for display purposes. $\Delta t_{0}$ is the time in the rigid state. The deformation stops at $t=t_{1}$. Heating pulse of $6.14 \mathrm{~J} \mathrm{~cm}^{-2}$. High voltage applied was $14.00 \mathrm{kV}$.

11 shows the photosignal and the heating pulse applied (scaled for display purposes). The photosignal remains at a constant value till $t=\Delta t_{0}$ where it starts to grow, reaches the maximum value, and stops at $t=t_{1}$. Figure 12 shows how the heating power modifies the duration of the fluid state. Curves (a), (b), and (c) correspond to underdevelopment, correct development, and overdevelopment, respectively. Humidity was $86 \%$ and ambient temperature $22^{\circ} \mathrm{C}$. The surface charge can be estimated using the $I-V$ characteristic of the corona device. ${ }^{22}$ We have determined $\Delta t_{0}$ to be of the order of $600 \mathrm{~ms}$ for a film of a global thickness equal to 55 $\mu \mathrm{m}$. Then our model should be applied to times greater than $\Delta t_{0}$.

The photosignal is related to the amplitude of the deformation, then we could test the influence of the electrostatic forces for a fixed heating power by considering sensitization

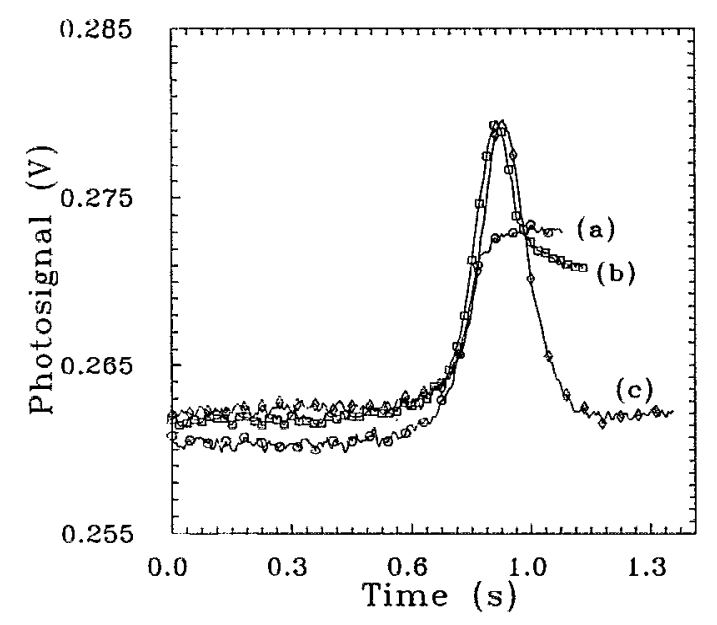

FIG. 12. Photosignal for different heating conditions. The heating pulses are (a) $5.46 \mathrm{~J} \mathrm{~cm}^{-2}$ (O), (b) $6.14 \mathrm{~J} \mathrm{~cm}^{-2}$ (口), and (c) $8.21 \mathrm{~J} \mathrm{~cm}^{-2}(\diamond)$. High voltage applied was $14.00 \mathrm{kV}$.

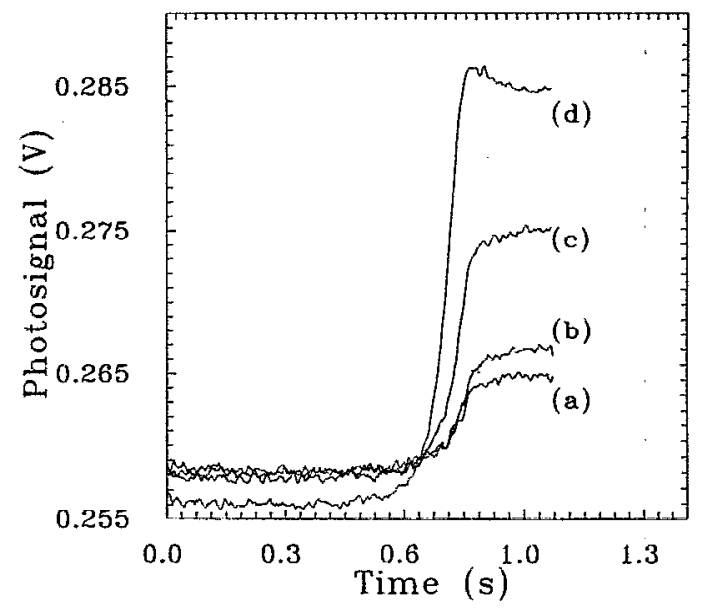

FIG. 13. Photosignal for different charging conditions. The heating was 6.14 $\mathrm{J} \mathrm{cm}^{-2}$ for all of them. The charging conditions were: (a) $13.90 \mathrm{kV}$; (b) $13.95 \mathrm{kV}$; (c) $14.10 \mathrm{kV}$; and (d) $14.25 \mathrm{kV}$.

of the film at various high voltages. Figure 13 shows clearly that the peak of the photosignal grows when the surface charge is augmented, indicating that the peak deformations have been increased.

\section{DISCUSSION AND CONCLUSIONS}

We outline and discuss the main hypothesis of the model presented in this article, as follows.

The thermoplastic in the fluid state is considered incompressible. This can be considered as a good approximation if the changes of the density due to temperature variations are negligible. In this way $\rho$ can be considered constant in Eq. (2.5). The viscosity $\eta$ of the fluid is also assumed to be independent of temperature: This has been shown to be accurate enough to predict the growth of the deformation (see Fig. 11). It should be pointed out that, in fact, the viscosity depends on temperature and molecular weight as is shown in Ref. 24 for a polystyrene film at temperatures and below $T_{g}$. A more complete approach to the problem of the frost deformation will take into account this effect and both the heat conduction and the velocity equations should be solved simultaneously (nonlinear coupled equations).

The convection term in Eq. (2.4) was neglected; then the model should be applied to a regime where the Reynolds number $\mathscr{B}$ is small compared to unity. The value of 98 remains in this range for all the cases considered in this article (typical values are of the order of 0.01 ).

The electrostatic field distributions were obtained neglecting the dark conductivity of the photoconductive layer. This effect can be considered of second order in practical applications. The photoconductive layer is also considered to have an infinite viscosity that, in practical terms, means that its glass transition temperature is far enough from the $T_{g}$ of the thermoplastic layer. Usually both the thermoplastic and the photoconductive layer have a common polymeric matrix but are doped with different amount of dyes, giving different values of $T_{g}$ for each layer. ${ }^{25}$ 
The optical properties of the thermoplastic layer, mainly the refraction index, are supposed to remain unmodified after heating and cooling the thermoplastic layer. This hypothesis has not been tested experimentally but it is implicitly assumed elsewhere. ${ }^{25}$

It is worthwhile to note that general surface displacements different from that given by Eq. (2.1) can be analyzed using Fourier techniques due to the linear character of Eq. (2.5). In the same way, general surface charge distributions may be considered as Eq. (5.2) is also linear.

In the dynamical model of the frost deformation of thermoplastic-photoconductor films presented in this article, the amplitude of the deformation is found to depend mainly on the thermoplastic layer thickness, the surface charge density, the permittivity of the photoconductor layer, the surface tension, and the rheological parameters of the thermoplastic. We have employed electrostatic potential distributions that are valid for the three-layer structure considering a sinusoidal charge distribution, showing that the involved electrostatic forces play a fundamental role in the dynamics of the deformation.

We have provided experimental evidence of the validity of the model for the frost deformation and the influence of the electrostatic field distribution measuring the scattered light by the film during heating. We have also measured with an AFM the spatial roughness of the film after the complete process (charging, heating, and cooling), given a measure of the peak deformation.

The theoretical model presented in this article provides a deep physical insight into the dynamics of the deformation of the photoconductor-thermoplastic film.

\section{ACKNOWLEDGMENTS}

We are especially indebted to L. L. Sánchez-Soto and J. M. Zoido for their useful comments and suggestions. We want to express our gratitude to $D$. Garrido who provided us support to do AFM measurements. We also acknowledge R. Yagüe for her manuscript review. This work has been carried out with the financial support of BRITE Project BE 3599 BRITE 0120-C of the EEC and MAT91-1389-CE.

\section{APPENDIX: ELECTROSTATIC FIELD DISTRIBUTIONS IN A THREE-LAYER FILM}

The study of electrostatic fields is of major importance for understanding the dynamics and the image formation characteristics of photoconductor-thermoplastic films. Neugebauer ${ }^{26}$ developed a method based on virtual charges to analyze the effects of electrostatic forces in xerographic devices. A different approach to the same problem is due to Schaffert. ${ }^{22}$ We have analyzed the electrostatic field distributions for a three-layer film ${ }^{27}$ and for completeness we reproduce here the main steps of the analysis.

The film is sensitized using a corona device, usually a positive corona, thus a surface charge distribution is achieved at the upper surface of the thermoplastic and its image at the top of the photoconductor. We will consider a sinusoidal distribution of charges on the upper surface of the photoconductive layer given by

$$
\bar{\sigma}=\frac{\sigma_{0}}{2}[1+\cos (k x)] .
$$

Note that $\sigma_{0}$ is related to surface charge distribution while $\sigma$ of Eq. (4.1) is the thermal conductivity of the thermoplastic layer.

The geometry considered is a three layer film in air: a substrate (A) the photoconductor (B), and the thermoplastic layer itself (C). The dielectric constants are $\epsilon_{a}, \epsilon_{b}$, and $\epsilon_{c}$, and $\epsilon_{d}=1$ is considered for the air. The Laplace equation must be solved to know the fields inside the different media involved,

$$
\nabla^{2} V=0
$$

We shall employ the ansatz to solve Eq. (A2),

$$
\begin{aligned}
V_{\alpha}(x, y)= & A_{\alpha}+A_{0 \alpha} y+\left[A_{1 \alpha} \exp (k y)\right. \\
& \left.+A_{2 \alpha} \exp (-k y)\right] \cos (k x),
\end{aligned}
$$

where $\alpha$ is a label for the regions from $\mathrm{A}$ to $\mathrm{D}, A_{\alpha}, A_{0 \alpha}$, $A_{1 \alpha}$, and $A_{2 \alpha}$ being constants to be determined by the boundary conditions for the potential and the electric field. ${ }^{28}$ The dark current of the photoconductive layer is neglected in the following analysis.

Solutions of the sinusoidal part can be treated separately from the linear term of Eq. (A3) for linear dielectric media. We are particularly interested in the solutions in regions $C$ and $\mathrm{D}$ that directly appear on the boundary problem associated with the fluid problem (3.8), that can be expressed as

$V_{\mathrm{C}}=C_{0}+C_{1} y\left[C_{1} \exp (k y)+C_{2} \exp (-k y)\right] \cos (k x)$,

$V_{\mathrm{D}}=D_{0}+D_{1} y\left[D_{1} \exp (k y)+D_{2} \exp (-k y)\right] \cos (k x)$.

After a lengthy calculation, the constants involved are found to be

$$
\begin{aligned}
C= & h_{0}\left(\frac{\epsilon_{b}}{\epsilon_{a}}-1\right) \frac{3 \sigma_{0}}{2 \epsilon_{b}}+h_{1} \frac{3 \sigma_{0}}{2 \epsilon_{b}}-h_{1} \frac{\sigma_{0}}{\epsilon_{b}}, \quad C_{0}=\frac{\sigma_{0}}{\epsilon_{c}} \\
D= & h_{0}\left(\frac{\epsilon_{b}}{\epsilon_{a}}-1\right) \frac{3 \sigma_{0}}{2 \epsilon_{b}}+h_{1} \frac{3 \sigma_{0}}{2 \epsilon_{b}}-h_{1} \frac{\sigma_{0}}{\epsilon_{b}}+\frac{\sigma_{0}}{\epsilon_{c}} h_{1}, \\
D_{0}=0 & \\
C_{1}= & \frac{\sigma_{0}\left(\epsilon_{c}-\epsilon_{d}\right)}{k D_{a}}\left[\epsilon_{a} \cosh \left(k h_{0}\right)-\epsilon_{b} \sinh \left(k h_{0}\right)\right] \\
& \times \exp \left(3 k h_{0}+k h_{1}\right)-\frac{\sigma_{0}\left(\epsilon_{c}-\epsilon_{d}\right)}{k D_{a}}\left[\epsilon_{a} \cosh \left(k h_{0}\right)\right. \\
& \left.+\epsilon_{b} \sinh \left(k h_{0}\right)\right] \exp \left(k h_{0}+3 k h_{1}\right),
\end{aligned}
$$




$$
\begin{aligned}
C_{2}= & \frac{\sigma_{0}\left(\epsilon_{c}+\epsilon_{d}\right)}{k D_{a}}\left[\epsilon_{a} \cosh \left(k h_{0}\right)-\epsilon_{b} \sinh \left(k h_{0}\right)\right] \\
& \times \exp \left(2 k h_{2}+3 k h_{0}+k h_{1}\right) \\
& -\frac{\sigma_{0}\left(\epsilon_{c}-\epsilon_{d}\right)}{k D_{a}}\left[\epsilon_{a} \cosh \left(k h_{0}\right)+\epsilon_{b} \sinh \left(k h_{0}\right)\right] \\
& \times \exp \left(2 k h_{2}+k h_{0}+3 k h_{1}\right),
\end{aligned}
$$

$$
\begin{aligned}
D_{1}= & 0, \\
D_{2}= & \frac{2 \sigma_{0} \epsilon_{c}}{k D_{a}}\left[\epsilon_{a} \cosh \left(k h_{0}\right)-\epsilon_{b} \sinh \left(k h_{0}\right)\right] \exp \left(2 k h_{2}\right. \\
& \left.+3 k h_{0}+k h_{1}\right)-\frac{2 \sigma_{0} \epsilon_{c}}{k D_{a}}\left[\epsilon_{a} \cosh \left(k h_{0}\right)\right. \\
& \left.+\epsilon_{b} \sinh \left(k h_{0}\right)\right] \exp \left(2 k h_{2}+k h_{0}+3 k h_{1}\right),
\end{aligned}
$$

where $D_{a}$ stands for

$$
\begin{aligned}
D_{a}= & 2\left[\epsilon_{a} \cosh \left(k h_{0}\right)-\epsilon_{b} \sinh \left(k h_{0}\right)\right]\left\{\left[\epsilon_{c}\left(\epsilon_{c}+\epsilon_{d}-\epsilon_{b}\right)-\epsilon_{b} \epsilon_{d}\right] \exp \left(2 k h_{2}+3 k h_{0}\right)+\left[\epsilon_{b} \epsilon_{d}-\epsilon_{c}\left(\epsilon_{c}+\epsilon_{b}-\epsilon_{d}\right)\right]\right. \\
& \left.\times \exp \left(3 k h_{0}+2 k h_{1}\right)\right\}+2\left[\epsilon_{a} \cosh \left(k h_{0}\right)+\epsilon_{b} \sinh \left(k h_{0}\right)\right]\left\{\left[\epsilon_{c}\left(\epsilon_{c}-\epsilon_{b}-\epsilon_{d}\right)+\epsilon_{b} \epsilon_{d}\right] \exp \left(k h_{0}+4 k h_{1}\right)\right. \\
& \left.-\left[\epsilon_{c}\left(\epsilon_{c}+\epsilon_{b}+\epsilon_{d}\right)+\epsilon_{b} \epsilon_{d}\right] \exp \left(2 k h_{2}+k h_{0}+2 k h_{1}\right)\right\}
\end{aligned}
$$

The electric field in regions $\mathrm{C}$ and $\mathrm{D}$ is straightforwardly calculated from the previous equations, obtaining

$$
\begin{aligned}
& E_{\mathrm{C} t}=-k\left[C_{1} \exp (k y)+C_{2} \exp (-k y)\right] \sin (k x), \\
& E_{\mathrm{C} n}=C_{0}+k\left[C_{1} \exp (k y)-C_{2} \exp (-k y)\right] \cos (k x), \\
& E_{\mathrm{D} t}=-k D_{2} \exp (-k y) \sin (k x), \\
& E_{\mathrm{D} n}=-k D_{2} \exp (-k y) \cos (k x) .
\end{aligned}
$$

It should be pointed out that the models employed by Budd $^{11}$ and Killat and Terrel ${ }^{13}$ are deduced for a single layer, the thermoplastic film; thus, the results presented here are of more general applicability.

${ }^{1}$ W. E. Glenn, J. SMPTE 69, 577 (1960).

${ }^{2}$ N. E. Woff, RCA Rev. 25, 200 (1964).

${ }^{3}$ E. C. Giaimo, RCA Rev. 25, 692 (1964).

${ }^{4}$ J. C. Urbach and R. W. Meier, Appl. Opt. 5, 666 (1966).

${ }^{5}$ J. Gaynor, IEEE Trans. Electron Devices ED-19, 512 (1972).

${ }^{6}$ T. C. Lee, Appl. Opt. 13, 888 (1974).

${ }^{7}$ L. H. Lin and H. L. Beauchamp, Appl. Opt. 9, 2088 (1970).

${ }^{8}$ J. Y. Moisan, P. Gravey, R. Lever, and L. Bonnel, Proc. Europ. Conf. Opt. 492, 51 (1984).

${ }^{9}$ J. C. Bellamy, D. B. Ostrowsky, M. Poindron, and E. Spitz, Appl. Opt. 10, 1458 (1971).
${ }^{10} \mathrm{G}$. Challa, Polymer Chemistry: An Introduction (Ellis Horwood, London, 1993).

${ }^{11}$ H. F. Budd, J. Appl. Phys. 36, 1613 (1965).

${ }^{12}$ U. Killat, J. Appl. Phys. 46, 5169 (1975).

${ }^{13}$ U. Killat and D. R. Terrel, Opt. Acta 24, 441 (1977).

${ }^{14}$ Z. Hirshfeld, A. A. Friesem, and Z. Rav-Noy, J. Appl. Phys. 52, 605 (1981).

${ }^{15}$ A. Handojo, J. Appl. Phys. 50, 886 (1979).

${ }^{16}$ D. W. Van Krevelen, Properties of Polymers (Elsevier, Amsterdam, 1972).

${ }^{17}$ L. D. Landau and E. M. Lifshitz, Fluid Mechanics (Pergamon, London, 1959).

${ }^{18}$ P. M. Morse and H. Feshbach, Methods of Theoretical Physics (McGrawHill, New York, 1953), Vol. 1.

${ }^{19}$ L. D. Landau and E. M. Lifshitz, Theory of Elasticity (Pergamon, London, 1959).

${ }^{20}$ R. J. Collier, C. B. Burckhardt, and J. L. Lin, Optical Holography (Academic, New York, 1971).

${ }^{21}$ J. A. Giacometti, J. Phys. D 20, 675 (1987).

${ }^{22}$ R. M. Schaffert, Electrophotography (Focal, London, 1980).

${ }^{23}$ W. T. Maloney and R. L. Gravel, Appl. Opt. 13, 2471 (1974).

${ }^{24}$ T. G. Fox, Jr. and P. J. Flory, J. Appl. Phys. 21, 581 (1950).

$25 \mathrm{~J}$. C. Urbach in Holographic Recording Materials edited by H. M. Smith (Springer, Berlin, 1977).

${ }^{26}$ H. E. J. Neugebauer, Appl. Opt. 3, 385 (1964).

${ }^{27} \mathrm{~F}$. Carreño and E. Bernabeu, in Proceedings of the 1st Annual Meeting EOS, 1993, Vol. 33, p. 147.

${ }^{28}$ J. D. Jackson, Classical Electradynamics (Wiley, New York, 1972). 
Journal of Applied Physics is copyrighted by the American Institute of Physics (AIP). Redistribution of journal material is subject to the AIP online journal license and/or AIP copyright. For more information, see http://ojps.aip.org/japo/japcr/jsp

Copyright of Journal of Applied Physics is the property of American Institute of Physics and its content may not be copied or emailed to multiple sites or posted to a listserv without the copyright holder's express written permission. However, users may print, download, or email articles for individual use. 
Journal of Applied Physics is copyrighted by the American Institute of Physics (AIP). Redistribution of journal material is subject to the AIP online journal license and/or AIP copyright. For more information, see http://ojps.aip.org/japo/japcr/jsp 